\title{
The Novel Paradigm of Stroke Mechanism Research Based on Neurovascular Unit
}

\author{
Qingshan Liü ${ }^{2, a, \#}$,Weiwei Zhang, ${ }^{2, b, \#}$, Xu Li ${ }^{2, c}$, Xiaoying Yin ${ }^{1, d,{ }^{*}}$ \\ 1Jiangxi University of Traditional Chinese Medicine, China \\ ${ }^{2}$ National Research Center for Minority Traditional Medicine, Minzu University of China, Beijing \\ 100081, China;

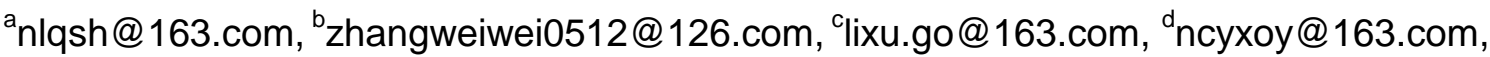 \\ ${ }^{\#}$ Weiwei Zhang and Qingshan Liu are co-first authors, *Yin Xiao-Ying is the corresponding author.
}

Keywords: Neurovascular unit; Stroke; Neurons; Blood-brain barrier (BBB);

\begin{abstract}
Objective: Stroke is a kind of disease with high morbidity, high mortality and high disability rate. Since currently available drugs treating cerebral ischemia have many limitations, it is necessary to find a new treatment approaches. The emergence of neurovascular unit provides a new perspective for basic research of stroke. Methods: Summarize the literature of research data relevant on the neurovascular unit and ischemia stroke, looking for the new treatment targets in the neurovascular unit during stroke process.Results: Checking out dozens of foreign literature, it shows that neurovascular unit plays an important role during the pathological and healing process of stroke. Conclusion: Neurovascular unit associated with the concept of the stroke into an integrated tissue reaction among all the cells and matrix components involved in the process of tissue damage, not just neurons or blood vessels involved in the pathological reactions. The concept will brought new opportunities for the treatment of stroke.
\end{abstract}

\section{Introduction}

Neurological disorders are highly prevalent around the globe. In 2008, neurodegenerative disorders were responsible for $1 \%$ of disabilities worldwide [1]. Strokes account for $55 \%$ of all neurological diseases and are considered the leading cause of permanent physical and mental disability [2]. Stroke affects the function and structure of blood-brain barrier, the loss of cerebral blood flow regulation, oxidative stress, inflammation and the loss of neural connections. Beyond thrombolysis for small subsets of cerebral ischemia patients, there are no therapies for stroke. The difficulty in drug development for stroke is perhaps best represented by the history of high-profile failures of a large number of neuroprotection clinical trials.

However, the emerging concept of the "neurovascular unit" suggests that focusing on saving neurons alone is not sufficient. The relevance of dynamic interactions between cerebral endothelial cells, astrocytes, pericytes and neurons is emphasized for brain function and dysfunction among the process of stroke. Neurovascular unit can be described as a "unit" which consists of blood brain barrier (BBB, endothelial cells, basal lamina matrix, astrocyte end-feet and pericytes), astrocytes, neurons, and their axons, in addition to other supporting cells (e.g. microglia and oligodendroglia)[3].The Neurovascular unit shows in Fig. 1. This provides a framework for considering bidirectional intercommunication between neurons and their neighboring supply microvessels via the intervening astrocytes. The resilience of the "unit" to reduction in flow or to flow cessation is unclear, but the processes are likely to be complex as adjacent units would be connected through their common microvessels and through dendritic connections. Furthermore, cell-cell interactions may be different than those perceived from isolated cell cultures. The concept of neurovascular unit associated with the stroke into an integrated tissue reaction among all the cells and matrix components involved in the 
process of tissue damage, not just neurons or blood vessels involved in the pathological reactions. The relevance of dynamic interactions between cerebral endothelial cells, astrocytes, pericytes and neurons is emphasized for brain function and dysfunction after stroke.

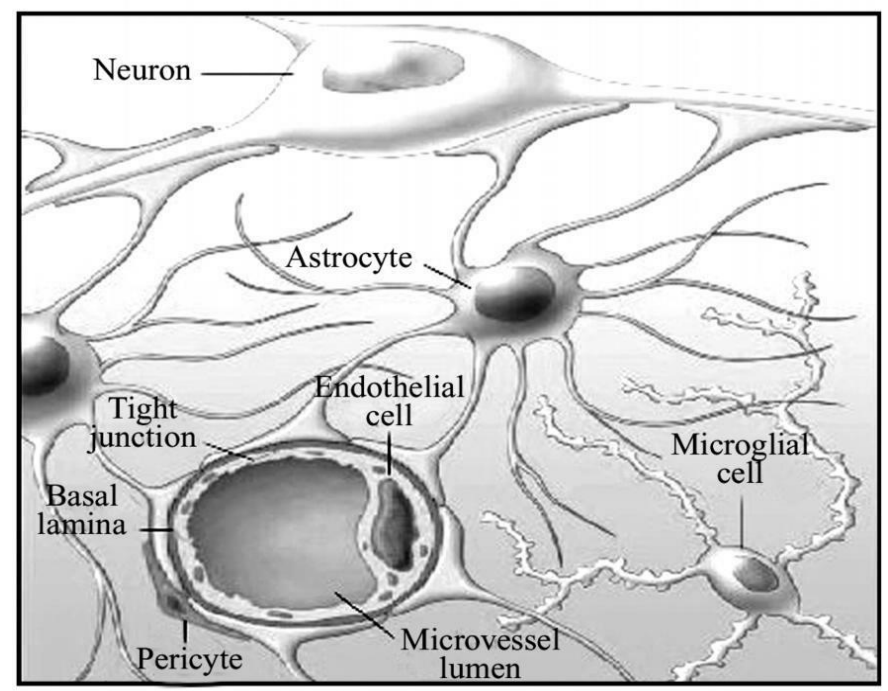

Fig. 1 The picture of neurovascular unit

\section{The Pathological process of ischemic stroke}

An interruption in the blood supply to the brain during ischemia results in oxygen-glucose deprivation and, consequently, reduced energy available for brain cell functions. In particular, neurons become incapable of maintaining the transmembrane ion gradients necessary for their function and homeostasis [4]. Then this even leads to excessive neuronal depolarization, an increase in the release of excitatory neurotransmitters and pro-inflammatory molecules, a reduction in the reuptake of these neurotransmitters from the extracellular space in penumbra and a GABAergic and dopaminergic misbalance in exo-focal areas [5]. Altogether, the pathological mechanisms induce an excessive intracellular accumulation of ions such as $\mathrm{Na}^{+}$and $\mathrm{Ca}^{2+}$ and, simultaneously, the deregulation of multiple signaling pathways, activating catabolic processes mediated by proteases, lipases, and nucleases, which interrupt neuronal function and induce cell death [6]. There is oxidative stress, excitotoxicity, energy metabolism and ion homeostasis mutual coupling with each other, which leads to ischemic cell death. The course of many these events suggests that, the processe highlighted here is not necessarily confined only to the vascular or to the extravascular compartments. They suggest that events that are initiated within the microvasculature often extend to other elements of the neuropil and involve glial and neuronal elements [7].

\section{The neurovascular unit in the development and regulation of stroke}

A growing database indicates that injury to neurons within the ischemic territory and dynamic changes in the supply microvasculature occur simultaneously in consequence of occlusion of a brain-supplying artery. The neurovascular unit is a hypothetical construct that links structurally and functionally the components of the cerebral microvasculature with their interdependent neurons via intervening astrocytes that are a component of individual microvessels. BBB breakdown is represented by the disruption of endothelial astrocyte-matrix interactions and leads to transmigration of inflammatory cells as well as toxic molecules into the brain parenchyma, which results in cerebral edema and hemorrhage. For the outer peripheral neutrophils play an important role in the BBB opening, able to penetrate the $\mathrm{BBB}$ damage, tissue damage caused by the release of toxic media. The outer peripheral neutrophils play an important role in the process of $\mathrm{BBB}$ opening, it can penetrate the damaged $\mathrm{BBB}$, 
cause tissue injury by releasing of toxic media. Microglia and the production of cytokine also aggravate the injury of BBB structure. Thus, tissue damage occurs by a series of events between blood microvascular - parenchymal cells after cerebral ischemia, eventually leads to the damage of neurons.

During cerebral development, microvessels and neurons are arranged relative to one another by growth along extracellular matrix paths. Astrocytes and endothelial cells interact to form the intervening basal lamina barrier and the inter-endothelial tight junctions as part of the permeability barrier of capillaries, and endothelial cells and astrocyte end-feet are required for the appearance of the permeability barrier phenotype. The BBB represents the key to maintaining an optimal brain microenvironment, which it is accomplished by restrictingmolecular passages to the brain from the systemic circulation. Astrocytes were once thought to play merely a housekeeping role in regards to neurons by providing them with structural and nutritional support. Today, increasing evidence has shown that astrocytes play a pivotal role in the development and regulation of the BBB, as well as in neuronal synapses, thereby making critical contributions to the maintenance of CNS homeostasis[8,9].

Currently, no gold standard treatments are available outside the acute therapeutic window to improve outcome in stroke patients. Some promising candidate targets have been identified for the improvement of long-term recovery after stroke, such as Rho GTPases, cell adhesion proteins, kinases, and phosphatases. Rho GTPases (Rac and RhoA) are involved in both tissue damage and survival, as these proteins are essential for the morphology and movement of neurons, astrocytes and endothelial cells, thus playing a critical role in the balance between cell survival and death [10]. Interestingly, neuroprotective agents, such as atorvastatin, and CDK5 silencing after cerebral ischemia and in a glutamate-induced excitotoxicity model may play an important role on the same cellular effectors to recover neurovascular unit integrity. The interaction of integrins with CNS extracellular matrix proteins helps guide astrocytes and their attendant mechanisms to positions ideally suited for maintaining the integrity of the BBB and the synapse during both developmental and adult stages. Integrins and connexins are among the most important molecules regulating astrocytic networks, an infrastructure that couples neuronal signaling and metabolic activity to the cerebral vascular tones, thereby balancing oxygen and energy demands with the supply of cerebral blood flow [11]. Astrocytopathy compromises the ability of astrocytes to maintain the homeostasis and integrity of neurovascular units, thereby undermining critical neuronal functions.

\section{Summary}

The functions of the neurovascular unit in neuropathology — both as a contributing factor to the development and progression of disease and in its responses to disease-indicate that the neurovascular unit is a therapeutic target [12]. In recent years, various gene knockout strategies in mice - most of which were notinitially aimed at studying neurovascular unit biology - have yielded the first insights about pathways important for neurovascular unit development and homeostasis. Neurovascular unit is a dynamic functional composite conceptual model, has difference in the structure and function because of the different positions and duration, will be a more realistic target for an ischemic injury. For any structural component of neurovascularunit injury unit will affect other components. Finally we believe that more studies are required in order to gain more insights in the interactions of every component within the neurovascular unit structure in health and disease. Large-scale mutagenesis screens in mice [13,14] will be necessary for identifying gene networks involved in neurovascular formation and function. These studies would be valuable to complete the puzzle, which will allow the development of novel therapeutic approaches based on neurovascular unit repair.

Those ongoing studies do not yet examine how these processes affect neuron excitability and membrane function. But, they do indicate at how many levels functions within the neurovascular unit framework are still not understood. One implication of this paucity of information and the clinical trial 
experienceso far is that interventions that solely target 'neuron protection' may be wholly inadequate toachieve clinical benefit. Strategies that extend to structural integrity and astrocyte functionwithin the neurovascular unit may be revealing. But, this will require a more fundamental understanding of the cell-cell, tissue functional, and neurovascular unit inter-relationships.

\section{Acknowledgement}

This work was financially supported by National Natural Science Foundation, PR China (81173657, 81273912, 81473424). Creative Item of Innovative team of Ministry of Education (IRT_13R63). Collaborative Innovation Center for the Modern Technology and Industrial Development of Jiangxi Minority Traditional Medicine Foundation （JXXT201403019）

\section{References}

[1] W.H.O. Global burden of disease communication. 2003. Available online at: http: // www.who.int/mental_health/neurology/en/.

[2] OMS. (2013).“Enferemedades cerebrovasculares Nota informativa”. Available online at: http://www.who.int/mediacentre/factsheets/fs317/es/.

[3] Gregory J. del Zoppo, M.D. Inflammation and the Neurovascular Unit in the Setting of Focal Cerebral Ischemia. Neuroscience. 2009, 158(3): 972-982.

[4] Szydlowska K and Tymianski M. Calcium, ischemia and excitotoxicity. Cell Calcium. 2010, 47(2): $122-129$.

[5] Sabogal AM, Arango CA, Cardona GP and Céspedes ÁE.. Atorvastatin protects GABAergic and dopaminergic neurons in the nigrostriatal system in an experimental rat model of transient focal cerebral ischemia. Biomedica. 2014, 34(2):207-217.

[6] Barreto GE1, Sun X, Xu L and Giffard RG. Astrocyte proliferation following stroke in the mouse depends on distance from the infarct. Plos One. 2011, 6(11): e27881.

[7] Maier CM, Hsieh L, Crandall T, Narasimhan P and Chan PH Ann Neurol. Evaluating therapeutic targets for reperfusion-related brain hemorrhage. 2006, 59(6):929-938.

[8] M. B'elanger, I. Allaman and P. J. Magistretti. Brain energy metabolism: focus on Astrocyte-neuron metabolic cooperation. Cell Metabolism. 2011, 14(6):724-738.

[9]C. Iadecola and M. Nedergaard. Glial regulation of the cerebral microvasculature. Nature Neuroscience.2007, 10(11):1369-1376

[10] Rafael Andres Posada-Duque, George E. Barreto, Gloria Patricia Cardona-Gomez1, et al. Protection after stroke: cellular effectors of neurovascular unit integrity. Front Cell Neurosci.2014, Published online 2014 Aug 14. doi: 10.3389/fncel.2014.00231.

[11] Hironobu Tanigami, Takayuki Okamoto, Yuichi Yasue and Motomu Shimaoka. Astroglial Integrins in the Development and Regulation of Neurovascular Units.2012,2012:1-10.

[12] Braint T. Hawkinds and Thomas P. Davis. The blood brain barrier / neurovascular unit in health and disease. Pharmacol Rev. 2005, 57:173-185.

[13] Ding S, Wu X, Li G, Han M, et al. Efficient transposition of the piggyBac (PB) transposon in mammalian cells and mice. Cell 2005, 122:473-483.

[14] Wu S, Ying G, Wu Q and Capecchi MR. Toward simpler and faster genome-wide mutagenesis in mice. Nat Genet 2007, 39:922-930. 